\title{
Regulation of fruit ripening by the brassinosteroid biosynthetic gene SICYP9OB3 via an ethylene- dependent pathway in tomato
}

\author{
Songshen Hu', Lihong Liu', Shuo Li', Zhiyong Shao', Fanliang Meng', Haoran Liu', Wenyi Duan', Dongyi Liang',
} Changqing Zhu', Tao Xu ${ }^{2}$ and Qiaomei Wang ${ }^{1}$

\begin{abstract}
The essential role of ethylene in fruit ripening has been thoroughly studied. However, the involvement of brassinosteroids (BRs) in the regulation of fruit ripening and their relationship with the ethylene pathway are poorly understood. In the current study, we found that BRs were actively synthesized during tomato fruit ripening. We then generated transgenic lines overexpressing or silencing SICYP90B3, which encodes a cytochrome P450 monooxygenase that catalyzes the rate-limiting step of BR synthesis. The expression level of S/CYP90B3 was positively related to the contents of bioactive BRs as well as the ripening process in tomato fruit, including enhanced softening and increased soluble sugar and flavor volatile contents. Both carotenoid accumulation and ethylene production were strongly correlated with the expression level of SICYP9OB3, corroborated by the altered expression of carotenoid biosynthetic genes as well as ethylene pathway genes in transgenic tomato fruits. However, the application of the ethylene perception inhibitor 1-methycyclopropene (1-MCP) abolished the promotion effect of SICYP90B3 overexpression on carotenoid accumulation. Taken together, these results increase our understanding of the involvement of SICYP90B3 in bioactive BR biosynthesis as well as fruit ripening in tomato, thus making SICYP90B3 a target gene for improvement of visual, nutritional and flavor qualities of tomato fruits with no yield penalty.
\end{abstract}

\section{Introduction}

Tomato (Solanum lycopersicum), an important agricultural crop species, is also a model system for studying fruit development and ripening. Fruit ripening in tomato is coordinated with brightened appearance caused by pigment accumulation; texture changes caused by tissue softening; and nutrient and flavor improvement through the bioactive compound, sugar, acid, and volatile organic compound (VOC) metabolism. The predominant pigments in mature tomato fruits are carotenoids, which

Correspondence: Tao Xu (syauxutao@syau.edu.cn) or

Qiaomei Wang (qmwang@zju.edu.cn)

${ }^{1}$ State Agricultural Ministry Laboratory of Horticultural Crop Growth and

Development, Department of Horticulture, Zhejiang University, Hangzhou 310058, China

${ }^{2}$ Key Laboratory of Protected Horticulture of Ministry of Education, College of Horticulture, Shenyang Agricultural University, Shenyang 110866, China

These authors contributed equally: Songshen Hu, Lihong Liu serve as antioxidants in defense against chronic diseases and can protect against some cancers ${ }^{1}$. Sucrose, fructose, and glucose are the most common sugars in ripening fruits $^{2}$. Volatiles are derived mainly from fatty acids, aliphatic amino acids, phenolic compounds, and carotenoids, including hydrocarbons, alcohols, aldehydes, esters, ethers, ketones, phenols, and sulfur- and nitrogencontaining compounds ${ }^{3,4}$. Among them, carotenoidderived volatiles is usually found to exert positive effects on tomato flavor and consumer preference ${ }^{5}$. Carotenoid cleavage dioxygenase 1 (CCD1) contributes to the formation of volatiles, including $\beta$-ionone, geranylacetone, 6 methyl-5-hepten-2-one (MHO), and pseudoionone, in tomato fruits ${ }^{4,6}$. Long-term tomato breeding manipulation, such as domestication, improvement, divergence, and introgression ${ }^{7}$, tends to weaken alleles involved in aromatic volatile biosynthesis and strengthen alleles

\section{(c) The Author(s) 2020}

(c) (i) Open Access This article is licensed under a Creative Commons Attribution 4.0 International License, which permits use, sharing, adaptation, distribution and reproduction cc) in any medium or format, as long as you give appropriate credit to the original author(s) and the source, provide a link to the Creative Commons license, and indicate if changes were made. The images or other third party material in this article are included in the article's Creative Commons license, unless indicated otherwise in a credit line to the material. If material is not included in the article's Creative Commons license and your intended use is not permitted by statutory regulation or exceeds the permitted use, you will need to obtain permission directly from the copyright holder. To view a copy of this license, visit http://creativecommons.org/licenses/by/4.0/. 
related to yield, disease resistance, and firmness ${ }^{8}$. Consequently, a reduction in the flavor of commercially produced tomato fruits has become a common consumer dissatisfaction. Many researchers have attached greater importance to the necessity and potential of improving fruit organoleptic and nutritional qualities in recent years ${ }^{9}$. Physiological changes in these complex biochemical characteristics during fruit ripening and quality formation occur in a highly synchronized manner and are regulated by the interaction of various factors, including external environmental stimuli and endogenous phytohormones ${ }^{10}$.

The onset of climacteric fruit ripening is accompanied by a burst of ethylene production that occurs in an autocatalytic manner ${ }^{11}$. Accordingly, ethylene acts as a master regulator that initiates and determines normal ripening of climacteric fruit ${ }^{12-14}$. Techniques manipulating ethylene biosynthesis, perception, or signal transduction are considered to be important strategies for modulation of fruit quality. In addition to ethylene, phytohormones such as auxin, gibberellin, abscisic acid, jasmonic acid, and brassinosteroids (BRs) have also been demonstrated to regulate carotenoid accumulation during tomato fruit ripening ${ }^{10}$. However, the molecular mechanisms of these phytohormones as well as their crosstalk in fruit ripening and quality formation have been less studied than those of ethylene.

BRs, which comprise various kinds of polyhydroxylated steroidal phytohormones, play broad roles in plant growth and development, including stomatal development, root growth, stem elongation, leaf epinasty, vascular differentiation, and floral development as well as in plant responses to abiotic and biotic stresses ${ }^{15}$. Extensive studies in Arabidopsis with molecular genetic approaches have revealed BR biosynthetic pathways and signaling pathways from receptor kinases in the plasma membrane to transcription factors in the nucleus. However, little attention has been paid to the regulatory role of BRs in tomato fruit ripening. BR treatment of tomato fruit pericarp discs enhances lycopene content as well as ethylene production ${ }^{16}$. Our previous study showed that ectopic overexpression (OE) of transcription factor genes (brassinazole resistant 1-1D, Atbzr1-1D), essential components of BR signaling, enhanced carotenoid accumulation in tomato fruits ${ }^{17}$. To date, more than 70 natural BRs have been identified in plants ${ }^{18}$, among which castasterone (CS) and brassinolide (BL) are frequently identified as being widely distributed and are considered to be the most bioactive BRs in plants ${ }^{19}$. However, BL has not been detected in tomato seedlings, demonstrating the presence of unique BR metabolic pathways as well as a potential role of $\mathrm{BL}$ in tomato at the reproductive stage ${ }^{20}$. Unlike other phytohormones, BRs function locally and display activity at very low concentrations (nanomolar to the picomolar range), and the physiological and biochemical effects of exogenous BR application are often concentration dependent. The spatiotemporal biosynthesis of BRs is usually strictly altered to maintain proper plant growth and development. To verify the mechanism by which BRs regulate fruit ripening, we need to pay more attention to the endogenous BR biosynthetic pathway in tomato fruits. There are two common parallel biosynthetic routes for CS and BL production: the early and late C-6 oxidation pathways ${ }^{21,22}$. In the late $\mathrm{C}-6$ oxidation pathway, campestanol $(\mathrm{CN})$ is initially oxidized to 6deoxocathasterone (6-deoxoCT), which then is successively oxidized to generate 6-deoxoteasterone (6-deoxoTE), 6-deoxo-3-dehydroteasterone (6-Deoxo3DT), 6deoxotyphasterol (6-deoxoTY), 6-deoxocastasterone (6deoxoCS), and CS (Fig. 1a). CS is then catalyzed to BL in the last step. Most enzymes involved in the BR biosynthetic pathway are members of the cytochrome $\mathrm{P} 450$ (Cyp450) superfamily (CYP85A, CYP90A, CYP90B, CYP90C, CYP90D, and CYP724B), with the exception of the reductase de-etiolated 2 (DET2) ${ }^{23}$. SIDWARF (SICYP85A1), which catalyzes 6-the conversion of deoxoCS to CS, is the first identified Cyp450 within the tomato BR biosynthetic pathway, with the related deficient mutant $d^{x}$ containing no detectable CS or $\mathrm{BL}^{24}$. Studies of SIDWARF OE have demonstrated an important role of BRs in tomato vegetative growth ${ }^{20}$. However, limited information is available about the function of BR biosynthetic pathway genes in fruit ripening and quality improvement.

DWF4 (CYP90B/CYP724B) catalyzes the C22 $\alpha$ hydroxylation reaction in the early stage and acts as a rate-limiting enzyme in BR biosynthesis ${ }^{25}$. OE of DWF4 leads to increased vegetative growth in Arabidopsis ${ }^{26}$ and Brassica napus ${ }^{27}$. Moreover, the regulation of DWF4 expression affects plant architecture ${ }^{28}$, seed size, and weight $^{29}$ in rice. The present study aims to elucidate the function of DWF4 in tomato fruit ripening and quality formation. Fruit-specific SlCYP90B3 (Solyc02g085360) was identified in tomato as an ortholog of Arabidopsis DWF4 (Supplementary Fig. 1). SlCYP90B3-OE and SlCYP90B3-RNAi transgenic lines were generated, and a subset of fruit ripening traits was characterized. The fruit ripening process, carotenoid accumulation, and ethylene production were found to be modulated by the SlCYP90B3 expression level. Moreover, favorable flavor volatiles was also improved in the fruits of SlCYP90B3-OE lines with no penalty yield. These results help to reveal the involvement of BRs in the regulation of tomato fruit ripening and the potential for manipulating SlCYP9OB3 to obtain more attractive and healthier fruits for human consumption. 
A

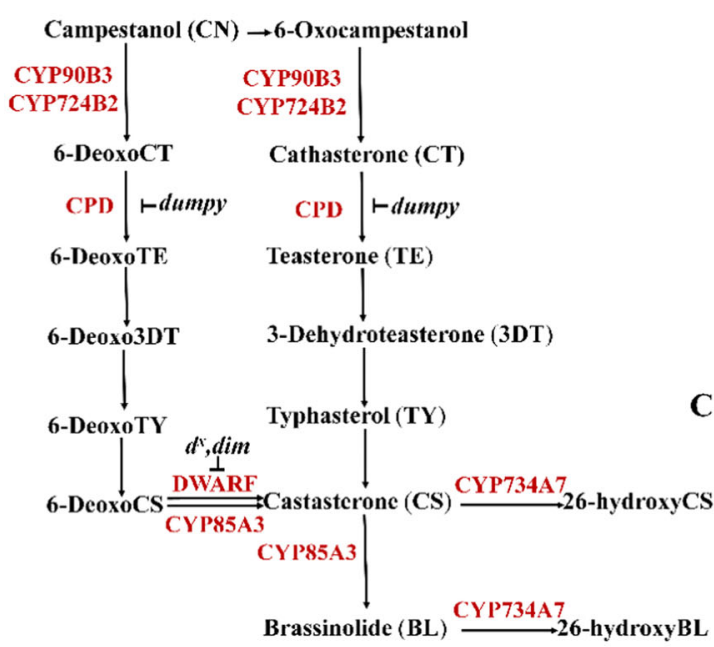

B

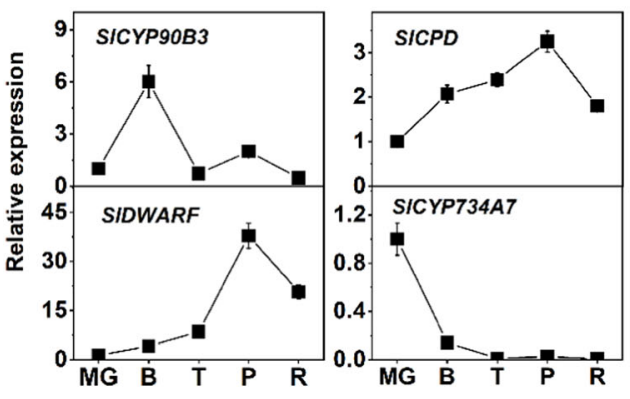

C

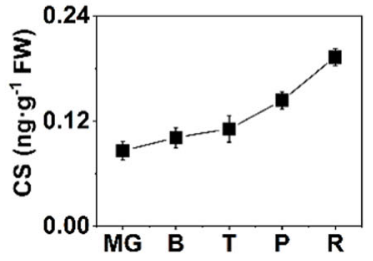

D

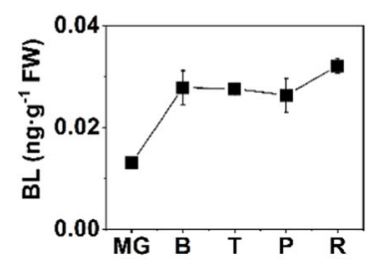

Fig. 1 Expression levels of BR biosynthetic and catabolic genes and changes in BR contents during tomato fruit ripening. a $B R$ biosynthetic and catabolic pathways in tomato. b Expression levels of BR biosynthetic genes (SICYP90B3, SICPD, SIDWARF) and the BR catabolic gene (SICYP734A7) during tomato fruit ripening. $\mathbf{c}$ Endogenous CS content during tomato fruit ripening. d Endogenous BL content during tomato fruit ripening. FW, fresh weight. Three biological replicates $(n=3)$ were used for each analysis. The error bars indicate the standard deviations. MG mature green, $\mathrm{B}$ breaker, T turning, P pink, R red.

\section{Results}

Expression of BR biosynthetic and catabolic genes and the accumulation of BRs during tomato fruit ripening

We examined the expression levels of BR biosynthetic genes (SlCYP90B3, SlCPD, SIDWARF) and catabolic genes (SlCYP734A7) during tomato fruit ripening (Fig. 1a). The expression level of $S l C Y P 90 B 3$ increased rapidly and peaked at the breaker stage (B), whereas the transcripts of $S I C P D$ and SIDWARF reached a peak at the pink stage (P) and then declined. In contrast, the expression of SlCYP734A7 decreased throughout fruit ripening (Fig. 1b). Next, we measured endogenous bioactive BR levels: CS and $\mathrm{BL}$ increased gradually from the mature green (MG) stage to the red stage (R) (Fig. 1c, d). These results imply that BRs are actively synthesized during tomato fruit ripening and may play a regulatory role in fruit ripening.

\section{Phenotypes of SICYP9OB3-OE and SICYP9OB3-RNAi transgenic tomato fruits}

Given that SICYP90B3 catalyzes the rate-limiting step in $\mathrm{BR}$ biosynthesis and that its transcript peaked at the breaker stage during fruit ripening (Fig. 1a, b), SlCYP90B3-OE and SlCYP90B3-RNAi constructs were introduced into tomato cultivar Ailsa Craig (AC). Two independent single-insert lines of both SlCYP90B3-OE and SlCYP90B3-RNAi were characterized after greenhouse cultivation for three successive generations. We noticed obvious color differences of the fruits at the $\mathrm{T}$ and P stages between the SlCYP90B3 transgenic lines and the wild type. Notably, the SICYP90B3-
$O E$ fruits at the $\mathrm{T}$ and $\mathrm{P}$ stages showed a stronger pigmentation phenotype according to visual observations (Fig. 2a). The expression levels of SlCYP90B3, SlCPD and SIDWARF increased in SICYP90B3-OE transgenic lines and decreased in the SICYP90B3-RNAi transgenic lines (Fig. 2b). To further verify whether endogenous levels of BRs were altered in the SICYP9OB3 transgenic plants, the BR contents were measured. The results showed that the levels of both $\mathrm{CS}$ and BL were significantly elevated in the SICYP90B3-OE transgenic lines but markedly decreased in the SlCYP90B3$R N A i$ transgenic lines (Fig. 2c, d).

Both SlCYP90B3-OE transgenic lines showed decreased fruit firmness at the $\mathrm{B}$ and $\mathrm{P}$ stages, while both SlCYP90B3-RNAi transgenic lines showed increased fruit firmness at the $\mathrm{P}$ and $\mathrm{R}$ stages (Fig. 3a). Compared with those in the wild-type fruits, the contents of total sugars in the SlCYP90B3-OE transgenic fruits were significantly higher at the $\mathrm{R}$ stage (Fig. $3 \mathrm{~b}$ ).

Volatile composition and content are desirable traits in tomato fruits and promote consumer palatability ${ }^{2,30}$. Twenty-eight-volatile compounds were detected in tomato fruits during ripening: 12 lipid-derived volatiles, 8 carotenoid-derived volatiles, 2 phenylalanine-derived volatiles, 3 phenylpropanoid-derived volatiles and 3 branched chain-derived volatiles (Fig. 3c, Supplementary Table 1). The total contents of the volatiles increased during fruit ripening, and compared with the wild type, the SICYP90B3-OE lines accumulated more volatiles at the MG, P, and R stages (Supplementary Fig. 2). 


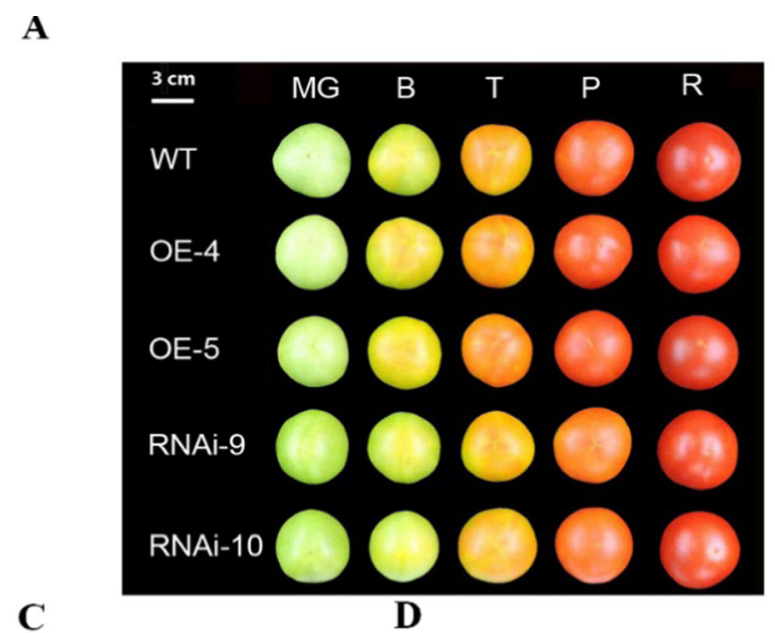

B
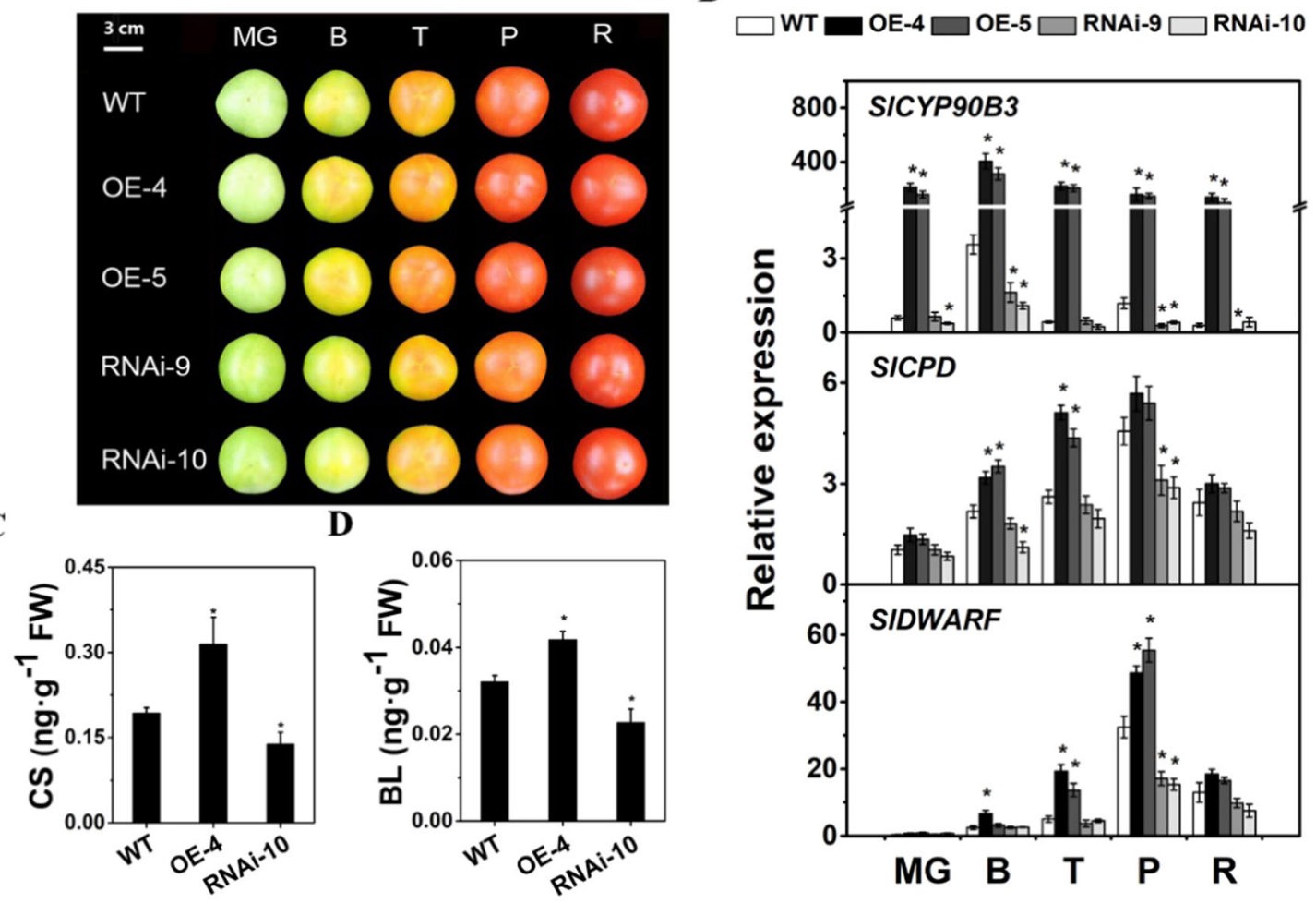

Fig. 2 Generation of SICYP90B3-OE and SICYP90B3-RNAi transgenic plants. a Fruits at five ripening stages from mature green to red. b Expression levels of BR biosynthetic genes during tomato fruit ripening. $\mathbf{c} C S$ content of fruits at the R stage. $\mathbf{d} B L$ content of fruits at the R stage. The scale bar represents $3 \mathrm{~cm}$. Three biological replicates $(n=3)$ were used for each analysis ( $P<0.05$; Student's $t$-test). The error bars indicate the standard deviations. WT wild type, MG mature green, B breaker, T turning, P pink, R red.

According to previous reports, carotenoid-derived volatiles are essential to tomato flavor and promote consumer palatability ${ }^{5,8}$, and their biosynthesis from carotenoids has been well studied (Fig. 3d). Geranylacetone and $\mathrm{MHO}$, which are catalyzed by oxidative cleavage of lycopene, are carotenoid-derived volatiles abundantly present in tomato fruits. The fruits of SlCYP90B3-OE lines at the $\mathrm{B}$ and $\mathrm{R}$ stages showed increased levels of $\mathrm{MHO}$ (Fig. 3e). Moreover, the content of geranylacetone also increased at the P stage in the SlCYP9OB3-OE lines compared with the wild type (Fig. 3e). However, the $\beta$-ionone level decreased at the R stage in the SLCYP90B3$O E$ fruits (Fig. 3e). CCD1B cleaves carotenoids to produce carotenoid-derived volatiles, which markedly increased at the B and P stages in the SlCYP9OB3-OE fruits (Fig. 3f). Taken together, these results suggest that increased carotenoid-derived volatiles caused by SlCYP90B3 overexpression may be due to enhanced expression of SlCCD1B.

SICYP90B3 OE promotes carotenoid accumulation in fruits

To determine the causes of the color changes of the fruits of the transgenic lines, the carotenoid (lycopene, $\beta$-carotene, and lutein) contents in the fruits of the SlCYP90B3 transgenic lines and the wild type at five typical developmental stages were analyzed. The level of lycopene significantly increased in both SLCYP9OB3-OE transgenic lines at all five stages, and the $\beta$-carotene level significantly increased at the $\mathrm{P}$ and $\mathrm{R}$ stages. In contrast, a significant decrease in lycopene content at the $\mathrm{B}, \mathrm{T}$, and $\mathrm{P}$ stages, along with a significant decrease in $\beta$-carotene content at the $\mathrm{P}$ and $\mathrm{R}$ stages, was observed in the two SlCYP90B3-RNA $i$ transgenic lines. A significant change in lutein content was observed only between the SLCYP90B3-RNA $i$ transgenic lines and the wild type at the B stage (Fig. 4a). The carotenoid biosynthetic pathway in tomato has been well elucidated. 1-Deoxy-D-xylulose-5phosphate synthase (DXS), geranylgeranyl pyrophosphate synthase (GGPPS), phytoene synthase 1 (PSY1), phytoene desaturase (PDS), $\beta$-carotene desaturase (ZDS) and $\beta$-cyclase (CYC-B) are the committed enzymes involved in carotenoid biosynthesis in tomato fruits. All five lycopene biosynthetic genes (SIDXS, SlGGPPS, SIPSY1, SlPDS, and $S I Z D S)$ presented the same expression patterns; their expression significantly increased in the SICYP90B3-OE transgenic lines and decreased in the SLCYP90B3-RNAi 
A

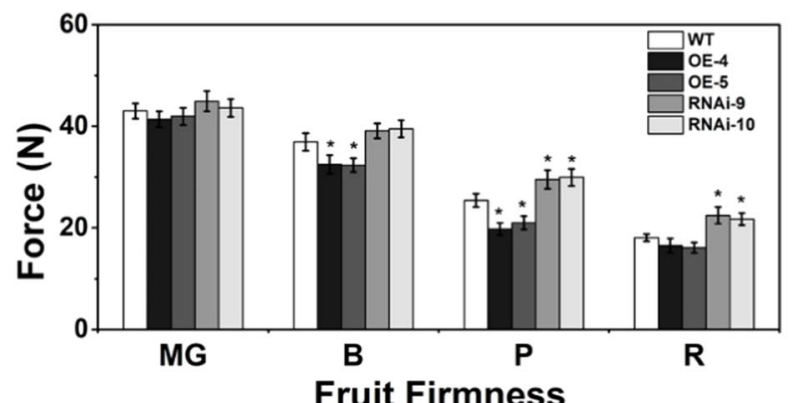

C Fruit Firmness

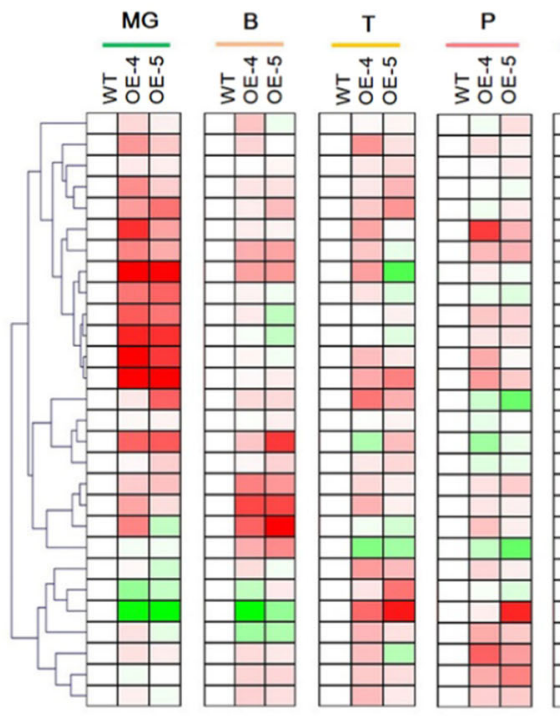

D

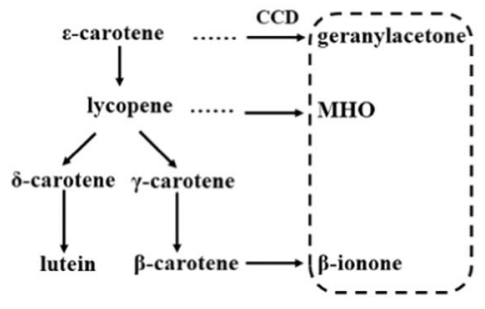

F
B

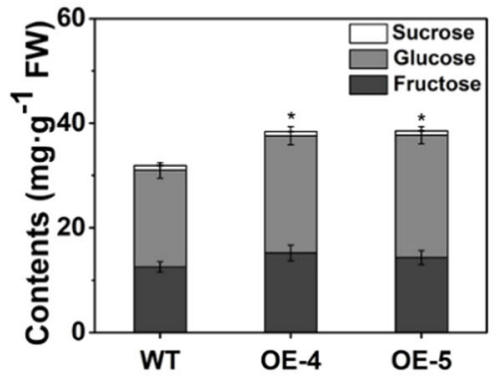

E
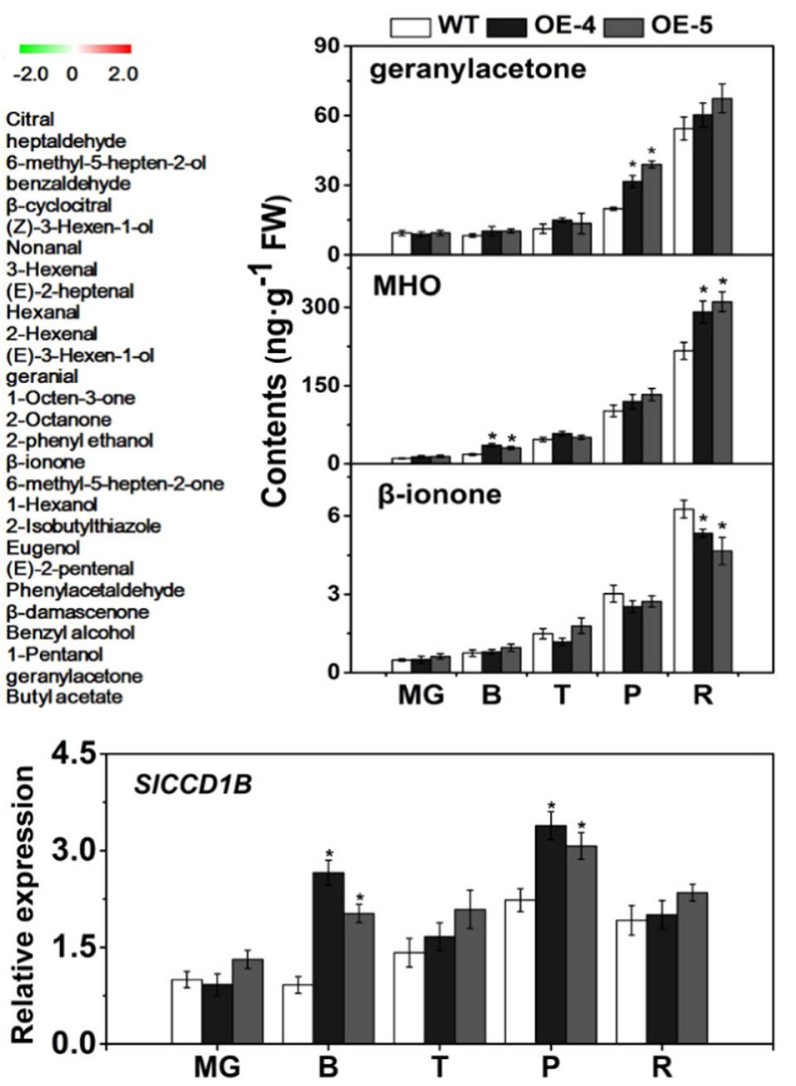

Fig. 3 Overexpression of SICYP9OB3 promotes tomato fruit ripening. a Fruit firmness at the MG, B, P and R stages. b Contents of soluble sugars in wild-type and SICYP9OB3-OE fruits at the R stage. $\mathbf{c}$ Heatmap visualization of volatiles in wild-type and S/CYP90B3-OE fruits during tomato fruit ripening. $\mathbf{d}$ Volatiles derived from carotenoids. e Contents of geranyl acetone, $\mathrm{MHO}$ and $\beta$-ionone during tomato fruit ripening. $\mathbf{f}$ The expression level of SICCDIB. Three biological replicates $(n=3)$ were used for each analysis $\left({ }^{*} P<0.05\right.$; Student's $t$-test). The error bars indicate the standard deviations. WT wild type, MG mature green, B breaker, T turning, P pink, R red.

transgenic lines (Fig. 4b). Among them, the expression levels of SIDXS, SIPDS, and SIZDS were elevated at the B and T stages in the SlCYP90B3-OE lines, and those of two other genes (SlGGPPS, SIPSY1) were significantly elevated at the MG, B, and T stages (Fig. 4b). The transcript level of SlGGPPS decreased at three sequential stages (B, T, and $\mathrm{P})$ in the SlCYP90B3-RNAi transgenic lines compared to the WT. The mRNA level of $S l C Y C-B$ increased at the P and R stages in the fruits of the SlCYP9OB3-OE transgenic lines. Moreover, the expression level of $S l C Y C-B$ significantly decreased at the $\mathrm{T}$ and $\mathrm{P}$ stages in the fruits of the SlCYP9OB3-RNAi transgenic lines (Fig. 4b). These results suggest that $\mathrm{OE}$ of $S l C Y P 90 B 3$ is related to increased carotenoid accumulation in tomato fruits. 
A

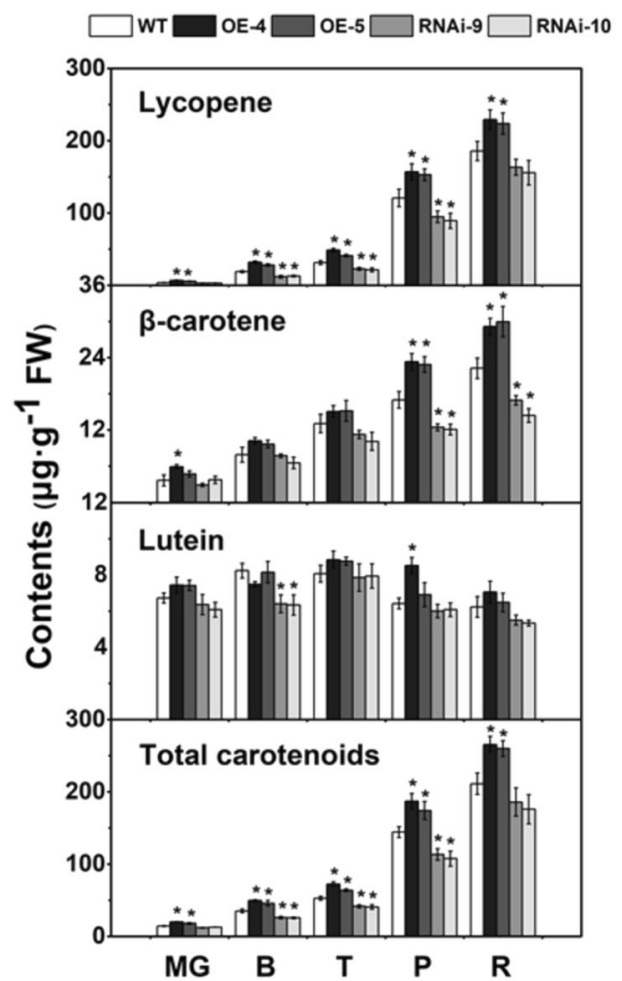

B

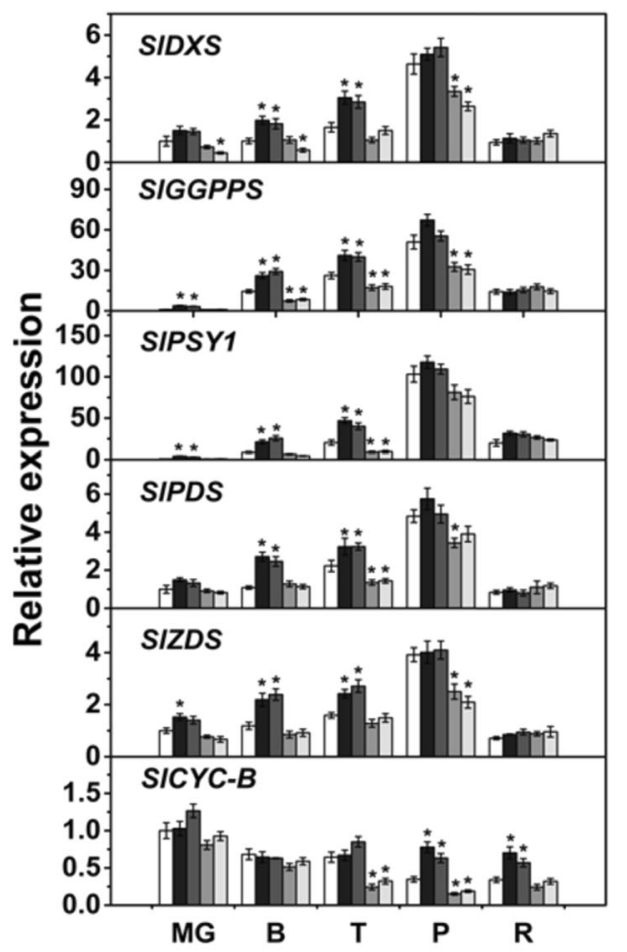

Fig. 4 Carotenoid contents and expression levels of carotenoid biosynthetic genes in wild-type, SICYP9OB3-OE, and SICYP90-RNAi transgenic fruits. a Carotenoid contents in tomato fruits. $\mathbf{b}$ Expression levels of carotenoid biosynthetic genes during tomato fruit ripening. Three biological replicates $(n=3)$ were used for each analysis ( ${ }^{*} P<0.05$; Student's $t$-test). The error bars indicate the standard deviations. WT wild type, MG mature green, B breaker, T turning, $\mathrm{P}$ pink, $\mathrm{R}$ red.

\section{OE of SICYP90B3 enhances ethylene production}

Ethylene is usually considered the master regulator of fruit ripening; thus, ethylene production was measured to verify the effects of SICYP90B3 on tomato fruit ripening. It was found that ethylene production increased at the B and $\mathrm{T}$ stages and peaked at the $\mathrm{P}$ stage in both the SlCYP90B3 transgenic lines and the wild type. Notably, higher ethylene content was observed in the SlCYP90B3$O E$ fruits at the $\mathrm{B}$ and $\mathrm{T}$ stages than in the wild-type fruits (Fig. 5a). These findings were consistent with elevated carotenoid levels and decreased firmness in the OE lines (Figs. 3a, 4a). We also examined the expression levels of ethylene biosynthesis and signaling genes and found that the expression of the ethylene biosynthetic genes (SIACS2, SlACS4, and SlACO1) significantly increased in the SlCYP90B3-OE transgenic lines at the MG, B and T stages (Fig. 5b). The expression levels of genes involved in ethylene signaling, including SIETR3 (encoding ethylene receptor) and SlCTR1 (encoding Raf-like kinase), also significantly increased (Fig. 5c). Similarly, ethylene response genes (SIE4, SIE8, and SIPG) were also upregulated in the SlCYP9OB3-OE transgenic lines at the MG, B and $\mathrm{T}$ stages (Fig. 5d). Taken together, these results suggest that OE of $S l C Y P 90 B 3$ enhanced both ethylene biosynthesis and signaling in tomato fruits.

\section{SICYP90B3 promotes carotenoid accumulation in an ethylene-dependent manner}

Here, we used 1-MCP, an effective ethylene perception inhibitor, to further explore the interaction between BRs and ethylene in promoting carotenoid accumulation. The contents of lycopene and total carotenoids increased in the SICYP90B3-OE fruits (OE-4) after $12 \mathrm{~d}$ and $18 \mathrm{~d}$ of storage compared with those in the wildtype fruits (Fig. 6), while no significant increase in lutein or $\beta$-carotene accumulation in the SICYP90B3-OE (OE4) fruits were observed after postharvest storage. The accumulation of lycopene and $\beta$-carotene was significantly repressed by $1-\mathrm{MCP}$ treatment at $6 \mathrm{~d}$ and $12 \mathrm{~d}$ of storage in both SICYP9OB3-OE and wild-type fruits, and the promotion of lycopene and total carotenoid accumulation in the SlCYP90B3-OE (OE-4) fruits were inhibited by 1-MCP treatment (Fig. 6). These results imply that SlCYP90B3 OE promotes carotenoid accumulation during tomato fruit ripening in an ethylenedependent manner. 
A

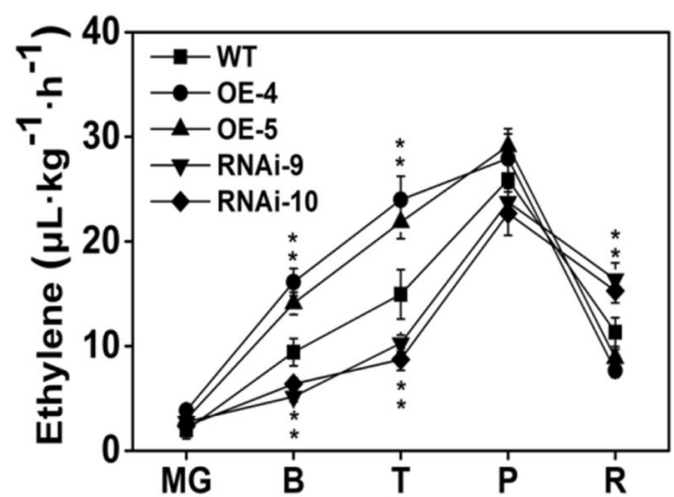

B

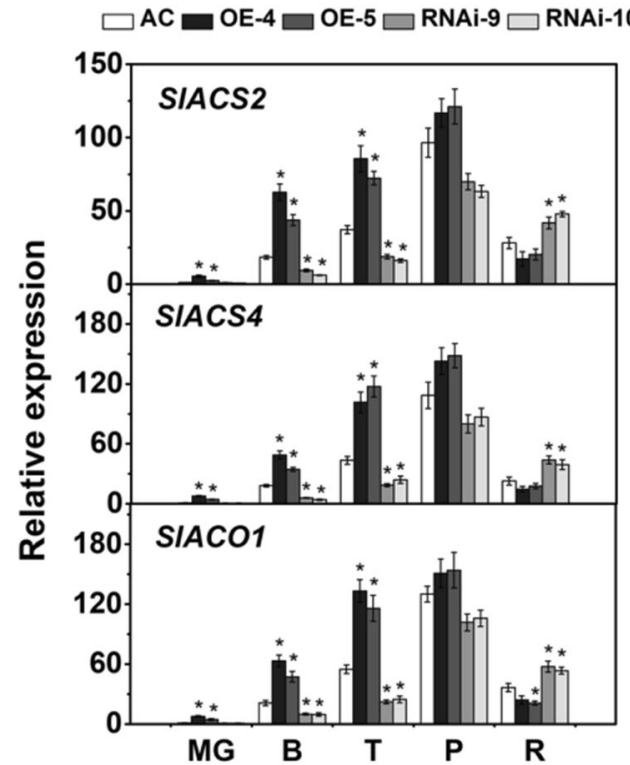

C

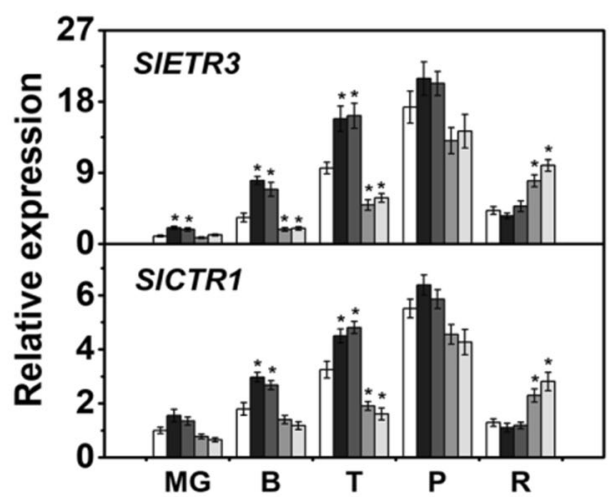

D

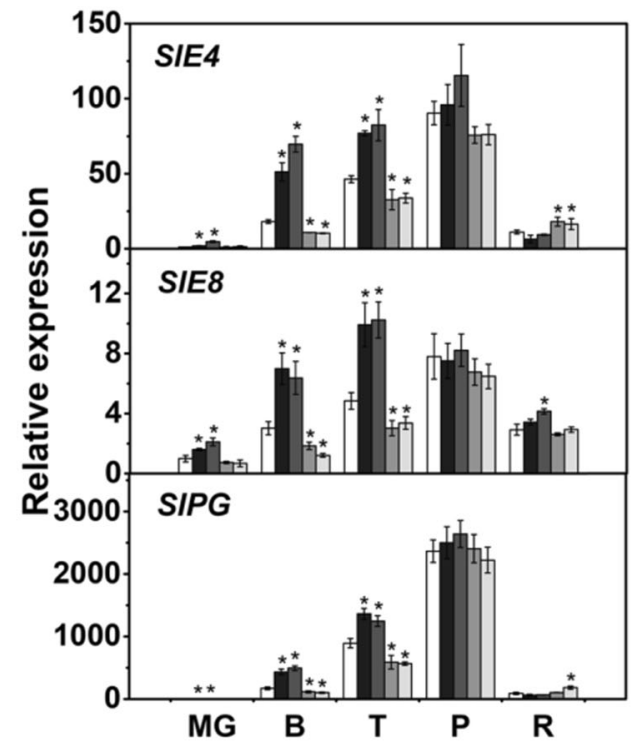

Fig. 5 Ethylene production and responses in SICYP9OB3-OE and SICYP90-RNAi transgenic fruits. a Ethylene production during tomato fruit ripening. b Expression levels of ethylene biosynthetic genes during tomato fruit ripening. ACS2, ACC synthase 2; ACS4, ACC synthase 4; ACO1, ACC oxidase 1; c Expression levels of genes involved in ethylene signaling during tomato fruit ripening. ETR3, ethylene receptor 3; CTR1, constitutive triple response 1. d Expression levels of ethylene response genes during tomato fruit ripening. E4, peptide Met sulfoxide reductase; E8, 1aminocyclopropane-1-carboxylate oxidase-like protein; $\mathrm{PG}$, polygalacturonase. Three biological replicates $(n=3)$ were used for each analysis $\left({ }^{*} P<\right.$ 0.05; Student's t-test). The error bars indicate the standard deviations. WT wild type, MG mature green, B breaker, T turning, P pink, R red.

\section{OE of SICYP90B3 improves tomato fruit yield}

After fruit quality evaluation, we measured the fruit yield of SlCYP9OB3-OE and SlCYP90B3-RNAi lines grown in the greenhouse and found that fruit development after pollination was accelerated in the SLCYP90B3-OE lines and delayed in the SlCYP90B3-RNAi lines in comparison with that in the wild type (Supplementary Fig. 3, Table 1). We then quantified the number of total fruits per plant at 135 days after sowing and found that the SLCYP90B3-OE plants produced more fruits than did the wild-type and SlCYP90B3-RNAi transgenic plants, but no significant difference in weight per fruit was detected among any of the transgenic lines or wild type. Moreover, the space required for the SICYP9OB3-OE transgenic plants decreased as a consequence of a decreased canopy; thus, the fruit yield of the OE- 4 and OE- 5 plants increased by $39.6 \%$ and $29.9 \%$, respectively, on an area basis. Thus, yield improvements could be achieved by the high-density planting of SlCYP9OB3-OE. These results indicate that SlCYP90B3 has the potential to improve both the quality and yield of tomato fruits.

\section{Discussion}

While increases in carotenoids and a sweet aroma during tomato fruit ripening are considered a wise means to promote seed dispersal, humans domesticate tomato as an important diet source with a focus on uniformity, size, yield, and shelf-life ${ }^{31}$. Currently, improvements in 


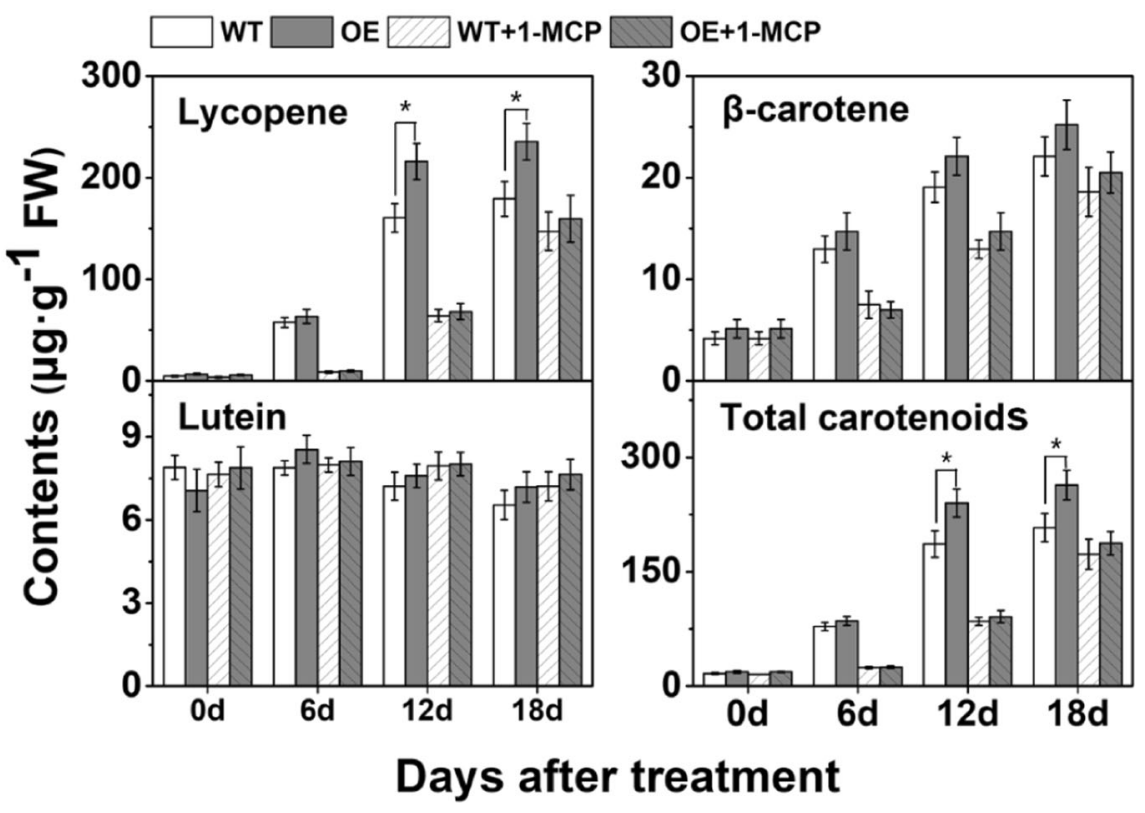

Fig. 6 Carotenoid accumulation in wild-type and SICYP9OB3-OE fruits with or without 1-MCP treatment. Here, $0.5 \mu L_{L} L^{-1} 1-M C P$ was applied as treatment. Three biological replicates $(n=3)$ were used for each analysis ( ${ }^{*} P<0.05$; Student's $t$-test). The error bars indicate the standard deviations.

Table 1 Fruit development and yield of wild-type and SICYP90B3 transgenic lines.

\begin{tabular}{lccccc}
\hline Parameter & WT & OE-4 & OE-5 & RNAi-9 & RNAi-10 \\
\hline Days from anthesis to fruit ripening & $52.4 \pm 1.7 \mathrm{~b}$ & $47.6 \pm 1.2 \mathrm{c}$ & $46.8 \pm 1.3 \mathrm{c}$ & $57.1 \pm 1.5 \mathrm{a}$ & $58.3 \pm 1.9 \mathrm{a}$ \\
Fruit number per plant & $18.4 \pm 1.6 \mathrm{~b}$ & $22.2 \pm 1.3 \mathrm{a}$ & $21.9 \pm 1.3 \mathrm{a}$ & $14.7 \pm 1.5 \mathrm{C}$ & $15.1 \pm 1.2 \mathrm{C}$ \\
Weight per fruit (g) & $29.7 \pm 5.8 \mathrm{a}$ & $25.9 \pm 5.5 \mathrm{a}$ & $26.7 \pm 4.4 \mathrm{a}$ & $34.6 \pm 6.2 \mathrm{a}$ & $35.9 \pm 5.1 \mathrm{a}$ \\
Fruit yield per plant (g) & $560.5 \pm 58.2 \mathrm{a}$ & $521.9 \pm 68.8 \mathrm{a}$ & $538.9 \pm 78.4 \mathrm{a}$ & $496.6 \pm 60.2 \mathrm{a}$ & $474.1 \pm 64.5 \mathrm{a}$ \\
Estimated fruit yield $\left(\mathrm{kg} \mathrm{m}^{-2}\right)$ & $4.12 \pm 0.44 \mathrm{~b}$ & $5.75 \pm 0.69 \mathrm{a}$ & $5.35 \pm 0.58 \mathrm{a}$ & $2.91 \pm 0.36 \mathrm{C}$ & $3.02 \pm 0.47 \mathrm{c}$ \\
\hline
\end{tabular}

The values shown are the means \pm SDs of 15 plants. The means denoted by the same letter do not differ significantly according to ANOVA in conjunction with Duncan's test $(P<0.05)$.

traditional organoleptic and functional characteristics in addition to external appearance are being reconsidered by breeders because consumers are paying increased amounts of attention to the flavor and health benefits of foods. A complete picture and functional annotation of the tomato genome, as well as genetic engineering, will not only offer improved potential but also accelerate the development of new crop varities ${ }^{32,33}$.

In this study, the contents of bioactive BRs, CS, and $\mathrm{BL}$, were found to gradually increase during tomato fruit ripening (Fig. 1c, d). Similarly, the presence of endogenous BRs at relatively high levels has been also been observed during the strawberry fruit-coloring period ${ }^{34}$ and at the onset of grape (Vitis vinifera) ripening ${ }^{35}$. The apparent BR accumulation in the fruits raises the possibility of a specific role of BRs in fruit ripening. To gain more insight into the regulatory mechanisms underlying fruit ripening, we manipulated the expression of SlCYP90B3 and analyzed its global effects on tomato ripening, including firmness, sugar content, flavor volatile contents, carotenoid accumulation, ethylene production, and yield. SlCYP90B3 OE in tomato fruit played a broad regulatory role in tomato improvement, which also included affecting fruit quality and yield. Manipulation of $S l C Y P 90 B 3$ with altered levels of bioactive BR contents and ethylene production in the current study helps to understand how BR gridlike biosynthetic routes are finely regulated and to understand the coordination with ethylene during tomato fruit ripening (Fig. 7). 
A

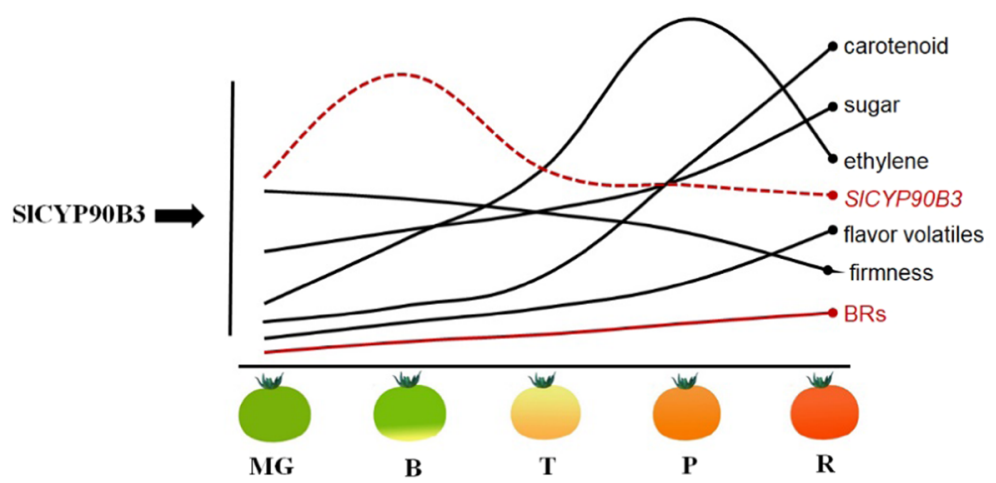

B

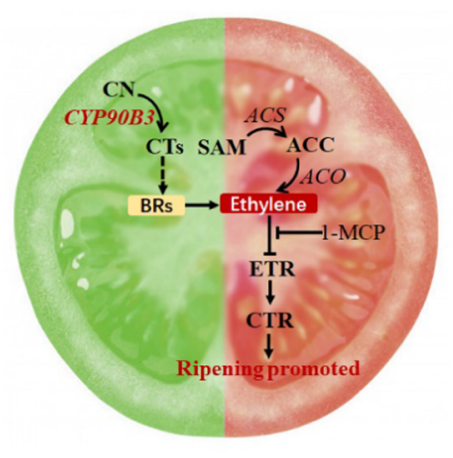

Fig. 7 A proposed model of the involvement of the BR biosynthetic gene SICYP90B3 in tomato fruit ripening in an ethylene-dependent manner. a During tomato fruit ripening, morphological and biochemical changes take place, including gradually elevated BR levels, sugar and carotenoid accumulation, enhanced release of ethylene and flavor volatiles, and reduced firmness. SICYP90B3 overexpression promotes all aspects of the ripening process. MG, mature green; $B$, breaker; $T$, turning; $P$, pink; $R$, red. $\mathbf{b}$ SICYP90B3 catalyzes the rate-limiting step from campestanol to cathasterone/6-deoxocathasterone in the BR biosynthetic pathway and increases the level of active BRs. The induced BRs in turn regulate the biosynthesis of ethylene and its downstream signaling to promote fruit ripening. CN campestanol, CTs cathasterone and 6-deoxocathasterone, BRs brassinosteroids, SAM S-adenosyl methionine, ACC 1-aminocyclopropane-1-carboxylic acid, ACS ACC synthase, ACO ACC oxidase.

\section{Fine tuning of BR biosynthesis during tomato fruit ripening}

Metabolic pathways in plants are interconnected and display interdependencies to enhance or suppress each other. BRs generally stimulate growth and development at low concentrations without active transportation, and plants are sensitive to excessive amounts of $\mathrm{BRs}^{15}$. Consequently, plants have evolved strategies to maintain BR homeostasis and normal function. The first strategy involves distinct developmental regulation. During vegetative growth, CS, but not $\mathrm{BL}$, was produced by SlCYP85A1 (SIDWARF), and CS exerts biological activity $^{24,36-38}$. SICYP85A3, which, interestingly, is expressed in fruits, catalyzes the same reaction as SICYP85A1 for CS production, while $\mathrm{BL}$ biosynthesis from CS was also detected $^{39}$. SlCYP724B2 and SICYP90B3 are functionally redundant in the early steps of BR biosynthesis in tomato. In immature fruits, only SlCYP90B3 mRNA was detected, and the expression level of the SlCYP90B3 transcript was higher than that of the SlCYP724B2 transcript in young leaves and mature fruits ${ }^{25}$. During fruit ripening, SlCYP90B3 expression was found to respond transiently at the B stage, whereas the mRNA levels of SlCPD and SIDWARF gradually increased from the B to P stages (Fig. $1 b)$. The second strategy involves feedback regulation. Exogenous BR treatment, as well as the increase in endogenous BR levels and active BR signaling, negatively regulates $\mathrm{BR}$ biosynthetic genes via a feedback loop ${ }^{40}$. Although the contents of endogenous $\mathrm{CS}$ and $\mathrm{BL}$ in $S I C Y P 90 B 3-O E$ fruits increased, the expression levels of $S I C P D$ and SIDWARF were upregulated rather than repressed (Fig. 2b-d). It could be that SICYP90B3 participates in the early step of BR biosynthesis and that the feedforward mechanism overcomes the feedback. The third strategy involves the deactivation or degradation of BRs. SICYP734A7 functions in BR catabolism in tomato ${ }^{41}$. The expression level of SlCYP734A7 dramatically decreased, affording increased levels of bioactive BRs (CS and $\mathrm{BL}$ ) during fruit ripening (Fig. 1b-d). The tactics within plants could call for a well-orchestrated modulation of BR biosynthesis and catabolism.

\section{BR and ethylene work together to promote carotenoid accumulation during fruit ripening}

OE of SlCYP90B3 was found to promote the accumulation of lycopene and $\beta$-carotene, as well as total carotenoids, via an orderly transcriptional regulation of genes involved in carotenoid biosynthesis (Fig. 4). Considering the central role of ethylene in fruit ripening, we investigated the influence of altered endogenous BR levels by manipulation of SICYP9OB3 on ethylene production (Fig. $5 \mathrm{a})$, as well as the expression of genes involved in ethylene biosynthesis, ethylene signaling, and the ethylene response (Fig. 5b-d). The results revealed that SlCYP90B3 OE enhanced both ethylene biosynthesis and signaling, which contributes to BR-enhanced carotenoid accumulation in tomato fruits. To further verify whether BRs regulate carotenoid accumulation in an ethylenedependent manner, we treated SlCYP90B3-OE transgenic fruits with $1-\mathrm{MCP}$, the most effective inhibitor of ethylene action, to rule out the involvement of ethylene. Surprisingly, the enhancement of carotenoid 
accumulation by SlCYP90B3 OE was eliminated by 1MCP treatment (Fig. 6), suggesting that BR-promoted carotenoid accumulation is ethylene dependent. In our previous work, we applied 3.0- $\mu \mathrm{M}$ EBL (2,4-epibrassinolide) to pericarp discs of a tomato ethylene receptor mutant, Never ripe (Nr) in the Pearson (PSN) background, and still observed an increase in the contents of lycopene and $\beta$-carotene in $\mathrm{Nr}$; the increase seemed to be ethylene independent ${ }^{17}$. The contrasting results between the application of exogenous BRs to the ethylene receptor mutant and the application of $1-\mathrm{MCP}$ to the SlCYP90B3-OE transgenic line are presumably due to the different conditions used. Additionally, the possibility that $\mathrm{Nr}$ plants retain residual ethylene responsiveness because of the partia1 dominance of the receptor gene (SlETR3) cannot be ruled out ${ }^{42}$. Interestingly, a large number of BR and ethylene coregulated genes have been detected in Arabidopsis, and these genes are enriched in BR-regulated BZR1 (an essential component of BR signaling) targets ${ }^{43}$. Moreover, BZR1 directly regulates several genes involved in ethylene biosynthesis and signaling ${ }^{43}$. A model by which the ethylene transcription factor EIN3 activates the MADS-box transcription factor RIN-TAGL1 to form a positive feedback loop amplifying autocatalytic ethylene synthesis has been illustrated ${ }^{44}$. The ripening process operates after the fruit reaches its final size at the MG stage, so the initial induction of ripening should be associated with both environmental and developmental cues. Both ethylene biosynthesis and signaling pathways are conserved in plants; thus, the BR-regulated BZR1 targets of ethylene biosynthetic genes should contribute to BR-promoted ethylene production in tomato fruits (Fig. 5). Moreover, SlCYP90B3 OE also shortened the tomato fruit growth period (Table 1). These results raise the possibility that BR functions via an ethylenedependent mechanism and that SlCYP9OB3 OE enhances ethylene-mediated ripening events in tomato fruit, including carotenoid accumulation, softening, and aromatic volatile emissions.

\section{Effects of SICYP9OB3 manipulation on tomato fruit softening}

Disassembly of cell wall components during ripening leads to fleshy fruit softening. The promotion of softening has been observed in exogenous EBL-treated climacteric fruits, including mango (Mangifera indica L. ${ }^{45}$ and persimmon (Diospyros kaki L.) ${ }^{46}$. Consistent with the effects of exogenous EBL-treatment, softening was also enhanced by SlCYP90B3 OE, with elevated BR levels, and was inhibited by $S l C Y P 90 B 3$ silencing, with reduced BR levels, in the present study (Fig. 3a). However, in both the EBLtreated mango and persimmon, as well as in tomato with elevated endogenous BR levels, ethylene production was promoted $^{45,46}$ (Fig. 5a), indicating an indirect role for BRs in climacteric fruit softening. However, the application of EBL to nonclimacteric grape ${ }^{35}$ and strawberry ${ }^{34}$ still significantly induced fruit ripening. In addition, BRs have been shown to activate several genes that encode cell walldegrading enzymes and that are BZR1 targets, such as polygalacturonase, pectinesterase, pectate lyase, endo-1,4$\beta$-glucanase, and $\beta$-galactosidase ${ }^{43}$. Overall, BR-regulated fruit softening may be achieved either by directly modulating cell wall degradation-related genes or by crosstalk with ethylene and other signaling pathways. Further research is needed to elucidate how BRs regulate fruit softening via their signaling and response components.

\section{SICYP90B3 OE improves flavor via modulation of volatile production}

The profile of each fruit flavor is unique, which determines its specific flavor. The limited knowledge of biosynthetic pathways and regulatory networks of flavorrelated volatiles is a challenge with respect to maintaining and improving flavor. Tomato volatiles are derived mainly from fatty acids, carotenoids and amino acids. Via applications of large consumer panels and analytical chemistry, a previous study identified 28 volatiles correlated with consumer liking ${ }^{8}$. The levels of 2-hexenal, 1-pentanol, and 1-hexanol from lipids; $\mathrm{MHO}$ and geranial from carotenoids; and 2-isobutylthiazole from branched-chain amino acids were elevated in SLCYP9OB3-OE tomato fruits (Supplementary Table 1). Among them, 1-pentanol, $\mathrm{MHO}$ and 2-isobutylthiazole contribute positively to consumer preferences ${ }^{2}$. The volatiles are active at nanomolar to picomolar concentrations, and even a slight increase in their content can lead to significant flavor enhancement. The tomato pangenome construction and analyses with phylogenetically and geographically representative accessions reveal that the positive regulation of apocarotenoid production contributes to desirable tomato flavor $^{33}$. Apocarotenoid volatiles derived from carotenoids through SICCD are typical in ripening tomato fruits (Fig. 3d). $S l C C D 1 B$, which accumulates throughout the ripening process ${ }^{6}$, encodes an enzyme that is more active than SlCCD1A is. In agreement with the elevated contents of geranylacetone and $\mathrm{MHO}$, the expression level of $S l C C D 1 B$ increased in response to $S l C Y P 90 B 3$ OE (Fig. $3 f)$. However, a reduction in $\beta$-ionone was observed in SlCYP90B3-OE tomato fruits at the R stage (Fig. 3e). This may have occurred because $\beta$-ionone is the substrate of other chemicals. Another possibility is that SICCD1B has different affinities for different substrates, and the metabolic flux to $\beta$-ionone accumulation could, therefore, be weakened. The enhanced transcription of $S l C C D 1 B$ might be attributed to increased BR levels or by the feedforward accumulation of carotenoids in SICYP90B3-OE fruits. 


\section{Experimental procedures}

\section{Plant materials and growth conditions}

Tomato (Solanum lycopersicum cv. Ailsa Craig) plants, including wild-type and two SlCYP90B3-OE (OE-4, OE-5) lines and SlCYP90B3-RNAi (RNAi-9, RNAi-10) lines, were grown in a greenhouse at $25 / 18{ }^{\circ} \mathrm{C}$ (day/night). The ripening stages ( $\mathrm{MG}, \mathrm{B}, \mathrm{T}, \mathrm{P}, \mathrm{R})$ of fruits were defined based on fruit color, as described previously ${ }^{47}$. There were three replicates per treatment, with six fruits per replicate. Fresh fruits were harvested to analyze the ethylene contents and firmness and then frozen in liquid nitrogen for subsequent RNA extraction and metabolite profiling.

\section{Transgenic plant construction}

SlCYP90B3-OE and RNAi constructs were generated using pGWB17 and pBIN19 vectors, respectively, as described previously ${ }^{48}$. For the construction of the $\mathrm{OE}$ construct, the full-length coding sequence of SlCYP90B3 was inserted downstream of the $35 \mathrm{~S}$ promoter in pGWB17 by Gateway cloning (Invitrogen, USA). For $R N A i$ vector construction, a $477 \mathrm{bp}$ fragment derived from the cDNA of SlCYP90B3 was synthesized via PCR and then fused into the plasmid pHANNIBAL in both antisense and sense orientations. The SlCYP90B3 RNA interference fragment was recombined to the plant binary vector pBIN19, generating SLCYP90B3-RNAi (Supplementary Table 2). The OE and RNAi vectors were then introduced into Agrobacterium tumefaciens strain LBA4404 (Tiangen, Beijing, China), and tomato transformation was performed as described ${ }^{49}$. Both independent OE (OE-4, OE-5) and RNAi (RNAi-9, RNAi-10) single-insertion lines were subsequently assessed after cultivation for three successive generations.

\section{RNA extraction and qRT-PCR-based analysis}

Total RNA was isolated from tomato fruits by the use of a TRIzol Reagent Kit (Takara Bio, Otsu, Japan), and 2\% $(\mathrm{w} / \mathrm{v})$ agarose gel was used to evaluate RNA integrity. One microgram of RNA was reverse-transcribed into cDNA using a PrimeScript cDNA Synthesis Kit (Takara, Japan). qRT-PCR was then performed using an iCycler (Bio-Rad, USA), and the gene-specific primers used are shown in Supplementary Table 3 . The relative gene expression was calculated according to the $2^{-\Delta \Delta C T} \operatorname{method}^{50}$.

\section{HPLC-MS/MS analysis of BRs}

The levels of bioactive endogenous BRs (CS and BL) were measured according to methods in a previous report $^{51}$. Approximately $2.0 \mathrm{~g}$ of tomato fruit flesh was ground into a powder in a precooled centrifuge tube and then extracted with $80 \%(\mathrm{v} / \mathrm{v})$ methanol. The mixture was shaken at $4{ }^{\circ} \mathrm{C}$ for $2 \mathrm{~h}$ and then centrifuged at 12,000 rpm for $10 \mathrm{~min}$ at $4{ }^{\circ} \mathrm{C}$. The supernatant was then eluted through prepacked Bond Elut (Agilent, USA), eluted with
$3 \mathrm{~mL}$ of methanol, eluted through a Strata-X membrane filter (Phenomenex, USA), eluted with $3 \mathrm{~mL}$ of methanol again and then evaporated to dryness under a nitrogenblowing instrument. Afterward, $200 \mu \mathrm{L}$ of methanol was added, and the solution was filtered through a $0.22 \mu \mathrm{m}$ membrane. Five microliters were subsequently subjected to HPLC-tandem mass spectrometry (HPLC-MS/MS) analysis. The contents of BRs were determined according to the concentration curve of BR standards (CS, BL; Sigma, USA).

\section{1-MCP treatment}

Sixty fruits of wild-type and SlCYP90B3-OE (OE-4) plants were harvested at the MG stage. For 1-MCP treatment, the fruits were placed in $25 \mathrm{~L}$ containers and treated with $0.5 \mu \mathrm{LL}^{-1} 1-\mathrm{MCP}$ for $16 \mathrm{~h}$. After 1-MCP treatment, the fruits were removed from the containers and stored at $25 \pm 1^{\circ} \mathrm{C}$.

\section{Measurement of ethylene production}

Ethylene production was detected according to the methods of a previous report ${ }^{52}$. Five fruits were enclosed in a $2.0 \mathrm{~L}$ sealed container and then incubated at $20^{\circ} \mathrm{C}$ for $1.5 \mathrm{~h}$, after which $1 \mathrm{~mL}$ of the headspace gas was collected and immediately injected into a gas chromatograph (Shimadzu GC-17A, Kyoto, Japan).

\section{HPLC analysis of carotenoids}

The extraction and analysis of carotenoids were performed as previously described via high-performance liquid chromatography (Shimadzu, Kyoto, Japan), with slight modifications ${ }^{52}$. Approximately $0.5 \mathrm{~g}$ of tomato fruit powder was extracted with $30 \mathrm{~mL}$ of hexane:acetone: ethanol $(1: 1: 1, \mathrm{v} / \mathrm{v} / \mathrm{v})$. The extracts were then centrifuged at $12,000 \mathrm{~g}$ for $15 \mathrm{~min}$, after which $15 \mathrm{~mL}$ of double distilled water was added. Three milliliters of the upper layer was eluted through a $0.22 \mu \mathrm{m}$ filter membrane and evaporated to dryness under a nitrogen-blowing instrument. The residue was then dissolved in $1.5 \mathrm{~mL}$ of tetrahydrofuran:acetonitrile:methanol (15:30:55, v/v/v), after which the solutions were loaded into a liquid phase bottle for HPLC analysis. The contents of the carotenoids were determined according to the concentration curve of standards (lutein, lycopene, and $\beta$-carotene; Sigma, USA).

\section{SPME-GC/MS analysis of volatiles}

The volatile contents were analyzed according to the methods of a previous report ${ }^{53}$. Five grams of fruit powder was put into a $10 \mathrm{~mL}$ headspace bottle, and then $5 \mathrm{~mL}$ of saturated $\mathrm{NaCl}$ solution and 2-octanol were added as internal standards. The samples were subsequently fully vortexed. The volatiles were extracted with a fiber coated with $50 / 30 \mu \mathrm{m}$ of divinylbenzene/carboxen/polydimethylsiloxane (DVB/CAR/PDMS) (Supelco, Bellefonte, 
PA, USA) for SPME-GC-MS analysis. The NIST 8.0 database (NIST/EPA/NIH, USA) was used to match the volatiles, and authentic standards were used to compare retention times. Quantification of the compounds was performed using the peak area of the internal standard as a reference based on the total ion chromatogram (TIC).

\section{Firmness determination}

Fruits were harvested from wild-type and transgenic lines at each developmental stage. Firmness was measured at three points on each fruit using a black plastic probe $(7.5 \mathrm{~mm})$ and a TA-XT2i texture analyzer (Stable Micro Systems Ltd., Godalming, UK). The puncture depth was set to $10 \mathrm{~mm}$, and the unit of firmness was measured in Newtons.

\section{Sugar content measurements}

The contents of glucose, sucrose, and fructose in tomato fruits were analyzed according to the methods of a previous report ${ }^{54}$. Approximately $0.1 \mathrm{~g}$ of tomato fruit powder was extracted in $1.5 \mathrm{~mL}$ of methanol and shaken at $950 \mathrm{rpm}$ for $15 \mathrm{~min}$ at $70^{\circ} \mathrm{C}$, followed by centrifugation at $11,000 \mathrm{rpm}$ for $10 \mathrm{~min}$. The supernatant was successively supplemented with $1.5 \mathrm{~mL}$ of double distilled water and $750 \mu \mathrm{L}$ of chloroform, after which the mixture was thoroughly vortexed. The internal standard ribitol was added, and the mixture was vacuum dried at room temperature. The derivatization process was as follows: $60 \mu \mathrm{L}$ of freshly prepared methoxyamine hydrochloride was added and shaken at $950 \mathrm{rpm}$ for $1.5 \mathrm{~h}$ at $37^{\circ} \mathrm{C}$; afterward, $40 \mu \mathrm{L}$ of NSTFA was added, and the solution was shaken at $950 \mathrm{rpm}$ for $0.5 \mathrm{~h}$ at $37^{\circ} \mathrm{C}$. The solutions were ultimately loaded into liquid phase bottles for GC analysis.

\section{Statistical analysis}

The experimental data were analyzed with SPSS 19.0 software. Pairwise comparisons were computed using Student's $t$-test $(P<0.05)$, while multiple comparisons were subjected to ANOVA using Duncan's test. Statistically significant differences $(P<0.05)$ are indicated by different lowercase letters.

The volatile data were normalized to the wild-type data with $\log _{2}$ transformation and visualized as a heatmap with hierarchical clustering performed by MultiExperiment Viewer 4.9 software.

\section{Acknowledgements}

This work was supported by the National Natural Science Foundation of China (Key Program, No. 31830078), the Ministry of Agriculture of China (2016ZX08009003-001), the Zhejiang Provincial Natural Science Foundation of China (No. LZ15C150001) and the Fundamental Research Funds for the Central University (2020QNA6023).

Conflict of interest

The authors declare that they have no conflict of interest.
Supplementary Information accompanies this paper at (https://doi.org/ 10.1038/s41438-020-00383-0).

Received: 6 May 2020 Revised: 16 July 2020 Accepted: 19 July 2020

Published online: 01 October 2020

\section{References}

1. Ford, N. A. \& Erdman, J. W. Jr. Are lycopene metabolites metabolically active? Biochim. Biophys. Acta 59, 1-4 (2012).

2. Klee, H. J. \& Tieman, D. M. The genetics of fruit flavour preferences. Nat. Rev. Genet. 19, 347-356 (2018).

3. Lewinsohn, E. et al. Enhanced levels of the aroma and flavor compound Slinalool by metabolic engineering of the terpenoid pathway in tomato fruits. Plant Physiol. 127, 1256-1265 (2001).

4. Klee, H. J. \& Tieman, D. M. Genetic challenges of flavor improvement in tomato. Trends Genet. 29, 257-262 (2013).

5. Vogel, J. T. et al. Carotenoid content impacts flavor acceptability in tomato (solanum lycopersicum). J. Sci. Food Agric. 90, 2233-2240 (2010).

6. Simkin, A. J. et al. The tomato carotenoid cleavage dioxygenase 1 genes contribute to the formation of the flavor volatiles beta-ionone, pseudoionone, and geranylacetone. Plant J. 40, 882-892 (2004).

7. Lin, T. et al. Genomic analyses provide insights into the history of tomato breeding. Nat. Genet. 46, 1220-1226 (2014).

8. Tieman, D. et al. A chemical genetic roadmap to improved tomato flavor. Science 355, 391-394 (2017).

9. Zhu, G. et al. Rewiring of the fruit metabolome in tomato breeding. Cell $\mathbf{1 7 2}$, 249-261 (2018).

10. Kumar, R., Khurana, A. \& Sharma, A. K. Role of plant hormones and their interplay in development and ripening of fleshy fruits. J. Exp. Bot. 65 4561-4575 (2014)

11. Rottmann, W. H. et al. 1-Aminocyclopropane-1-carboxylate synthase in tomato is encoded by a multigene family whose transcription is induced during fruit and floral senescence. J. Mol. Biol. 222, 937-961 (1991).

12. Srivastava, A. \& Handa, A. K. Hormonal regulation of tomato fruit development: a molecular perspective. J. Plant Growth Regul. 24, 67-82 (2005).

13. Argueso, C. T., Hansen, M. \& Kieber, J. J. Regulation of Ethylene Biosynthesis. J. Plant Growth Regul. 26, 92-105 (2007).

14. Barry, C. S. \& Giovannoni, J. J. Ethylene and fruit ripening. J. Plant Growth Regul. 26, 143 (2007).

15. Vukašinović, N. \& Russinova, E. Brexit: possible brassinosteroid export and transport routes. Trends Plant Sci. 23, 285-292 (2018).

16. Vardhini, B. V. \& Rao, S. S. R. Acceleration of ripening of tomato pericarp discs by brassinosteroids. Phytochemistry 61, 843-847 (2002)

17. Liu, L. et al. Ectopic expression of a BZR1-1D transcription factor in brassinosteroid signalling enhances carotenoid accumulation and fruit quality attributes in tomato. Plant Biotechnol. J. 12, 105-115 (2014).

18. Sharma, A. et al. Brassinosteroid-mediated pesticide detoxification in plants: a mini-review. Cogent Food Agric. 4, 1436212 (2018)

19. Bajguz, A. \& Tretyn, A. The chemical characteristic and distribution of brassinosteroids in plants. Phytochemistry 62, 1027-1046 (2003).

20. Li, X. J. et al. DWARF overexpression induces alteration in phytohormone homeostasis, development, architecture and carotenoid accumulation in tomato. Plant Biotechnol. J. 14, 1021-1033 (2016).

21. Suzuki, H., Kim, S. K., Takahashi, N. \& Yokota, T. Metabolism of castasterone and brassinolide in mung bean explant. Phytochemistry 33, 1361-1367 (1993).

22. Fujioka, S. \& Yokota, T. Biosynthesis and metabolism of brassinosteroids. Annu. Rev. Plant. Biol. 54, 137-164 (2003).

23. Ghosh, S. Triterpene structural diversification by plant cytochrome $\mathbf{P 4 5 0}$ enzymes. Front. Plant Sci. 8, 1886 (2017).

24. Bishop, G. J. et al. The tomato DWARF enzyme catalyses C-6 oxidation in brassinosteroid biosynthesis. Proc. Natl Acad. Sci. USA 96, 1761-1766 (1999)

25. Ohnishi, T, Watanabe, B. Sakata, K. \& Mizutani, M. CYP724B2 and CYP90B3 function in the early C-22 hydroxylation steps of brassinosteroid biosynthetic pathway in tomato. Biosci. Biotechnol. Biochem. 70, 2071-2080 (2006a)

26. Choe, S. et al. Overexpression of DWARF4 in the brassinosteroid biosynthetic pathway results in increased vegetative growth and seed yield in Arabidopsis. Plant J. 26, 573-582 (2001) 
27. Sahni, S. et al. Overexpression of the brassinosteroid biosynthetic gene DWF4 in Brassica napus simultaneously increases seed yield and stress tolerance. Sci. Rep. 6, 28298 (2016).

28. Wu, C. Y. et al. Brassinosteroids regulate grain filling in rice. Plant Cell 20, 2130-2145 (2008).

29. Sakamoto, T. et al. Erect leaves caused by brassinosteroid deficiency increase biomass production and grain yield in rice. Nat. Biotechnol. 24, 105-109 (2006).

30. Patrick, J. W., Botha, F. C. \& Birch, R. G. Metabolic engineering of sugars and simple sugar derivatives in plants. Plant Biotechnol. J. 11, 142-156 (2013).

31. Scossa, F. \& Fernie, A. R. How fruit ripening is ENCODEd. Nat. Plants 4, 744 (2018).

32. Zsögön, A. et al. De novo domestication of wild tomato using genome editing. Nat. Biotechnol. https://doi.org/10.1038/nbt.4272 (2018).

33. Gao, L. et al. The tomato pan-genome uncovers new genes and a rare allele regulating fruit flavor. Nat. Genet. 51, 1044-1051 (2019).

34. Chai, Y. M. et al. Brassinosteroid is involved in strawberry fruit ripening. J. Plant Growth Regul. 69, 63-69 (2013).

35. Symons, G. M., Davies, C., Shavrukov, Y., Dry, I. B. \& Thomas, M. R. Grapes on steroids. brassinosteroids are involved in grape berry ripening. Plant Physiol. 140, 150-158 (2006)

36. Nomura, T., Nakayama, M., Reid, J. B., Takeuchi, Y. \& Yokota, T. Blockage of brassinosteroid biosynthesis and sensitivity causes dwarfism in garden pea. Plant Physiol. 113, 31-37 (1997).

37. Koka, C. V. et al. A putative role for the tomato genes DUMPY and CURL-3 in brassinosteroid biosynthesis and response. Plant Physiol. 122, 85-98 (2000).

38. Shimada, Y. et al. Brassinosteroid-6-oxidases from Arabidopsis and tomato catalyze multiple C-6 oxidations in brassinosteroid biosynthesis. Plant Physiol. 126, 770-779 (2001)

39. Nomura, $T$. et al. The last reaction producing brassinolide is catalyzed by cytochrome P-450s, CYP85A3 in tomato and CYP85A2 in Arabidopsis. J. Biol. Chem. 280, 17873-17879 (2005).

40. He, J. X. et al. BZR1 is a transcriptional repressor with dual roles in brassinosteroid homeostasis and growth responses. Science 307, 1634-1638 (2005).
41. Ohnishi, T. et al. Tomato cytochrome P450 CYP734A7 functions in brassinosteroid catabolism. Phytochemistry 67, 1895-1906 (2006b).

42. Lanahan, M. B., Yen, H. C., Giovannoni, J. J. \& Klee, H. J. The never ripe mutation blocks ethylene perception in tomato. Plant Cell 6, 521-530 (1994).

43. Sun, $Y$. et al. Integration of brassinosteroid signal transduction with the transcription network for plant growth regulation in Arabidopsis. Dev. Cell. 19 765-777 (2010).

44. Lü, P. et al. Genome encode analyses reveal the basis of convergent evolution of fleshy fruit ripening. Nat. Plants 4, 784-791 (2018).

45. Zaharah, S. S., Singh, Z., Symons, G. M. \& Reid, J. B. Role of brassinosteroids, ethylene, abscisic acid, and indole-3-acetic acid in mango fruit ripening. J. Plant Growth Regul. 31, 363-372 (2012).

46. He, Y., Li, J., Ban, Q., Han, S. \& Rao, J. Role of brassinosteroids in persimmon (Diospyros kaki l.) fruit ripening. J. Agric. Food Chem. 66, 2637-2644 (2018).

47. Giovannoni, J. J. Genetic regulation of fruit development and ripening. Plant Cell 16, S170-S180 (2004).

48. Dong, T. et al. A tomato MADS-box transcription factor, SIMADS1, acts as a negative regulator of fruit ripening. Plant Physiol. 163, 1026-1036 (2013).

49. An, G. Binary ti vectors for plant transformation and promoter analysis. Methods Enzymol. 153, 292-305 (1987)

50. Livak, K. J. \& Schmittgen, T. D. Analysis of relative gene expression data using real-time quantitative PCR and the $2^{-\Delta \Delta C T}$ method. Methods 25, 402-408 (2001).

51. Pan, G. et al. Brassinosteroids mediate susceptibility to brown planthopper by integrating with salicylic acid and jasmonic acid pathways in rice. J. Exp. Bot. 69, 4433-4442 (2018)

52. Liu, L. et al. Ethylene independent induction of lycopene biosynthesis in tomato fruits by jasmonates. J. Exp. Bot. 63, 5751-5761 (2012).

53. Wang, L. et al. Effect of methyl salicylate and methyl jasmonate pre-treatment on the volatile profile in tomato fruit subjected to chilling temperatures. Postharvest Biol. Technol. 108, 28-38 (2015).

54. Lisec, J., Schauer, N., Kopka, J., Willmitzer, L. \& Fernie, A. R. Gas chromatography mass spectrometry-based metabolite profiling in plants. Nat. Protoc. 1, 387-396 (2006). 